\title{
Irukandji-like syndrome caused by single-tentacle box jellyfish found in Thailand, 2007-2019
}

\author{
Lakkana Thaikruea $\mathbb{}$
}

Department of Community Medicine, Faculty of Medicine, Chiang Mai University, Thailand

\begin{abstract}
Background: Irukandji syndrome definition is still widely misunderstood. Irukandji-like syndrome is more unclear than Irukandji syndrome. This study aimed to describe Irukandji-like syndrome in cases involving stinging by single-tentacle box jellyfish species in Thailand.

Materials and methods: Surveillance system and networks of toxic jellyfish incidents were established to enable case detection. In the period 2007 to 2019, all cases of stinging by single-tentacle box jellyfish resulting in collapse, hospital attendance or death were investigated.

Results: The majority of the 19 Irukandji-like syndrome cases were male (68.2\%), median age 35.0 years (range 6.0-60.0), and Thai nationality (52.3\%). Clinical manifestations of Irukandji-like syndrome were categorised as severe wound pain with immediate systemic reaction (66.7\%), moderate wound pain with gradual systemic reaction (16.7\%), and moderate wound pain with the immediate systemic reaction after a physical/chemical trigger (16.7\%). The pain occurring when being stung differed from the pain occurring during the systemic reaction. The five most common symptoms were pain (100.0\%), high blood pressure (100.0\%), palpitations (86.7\%), respiratory distress (52.6\%), and near collapse/collapse (31.6\%). The pain occurs when being stung was excruciating or burning pain at the wounds, felt like an electric shock, and rapidly expanded to heart pain. While the pain occurring during the systemic reaction was back pain, muscle pain, joint pain, abdominal pain, and body aches. The marks from the tentacles appeared similar in appearance to the caterpillar tracks of tanks. In 6 cases the species could be identified and all of them involving the Morbakka spp.

Conclusions: This was the largest study of Irukandji-like syndrome cases involving stings by single-tentacle box jellyfish in Thailand and the different clinical manifestations might be caused by different species of single-tentacle box jellyfish.
\end{abstract}

(Int Marit Health 2020; 71, 2: 91-96)

Key words: Irukandji-like syndrome, box jellyfish, envenomation, sign, symptom, definition

\section{INTRODUCTION}

The occurrence of box jellyfish envenomation was initially denied in Thailand. In 2008 a medical epidemiologist from the Ministry of Public Health and one from the Faculty of Medicine, Chiang Mai University (author) started to investigate this issue and provided evidence to show the existence of lethal box jellyfish envenomation in Thailand [1-3]. Dangerous envenomation incidents concerning box jellyfish in Thailand have involved both multiple-tentacle and single-tentacle box jellyfish. Multiple-tentacle box jellyfish, Chironex spp. have caused fatal and near-fatal cases [4-10]. Single-tentacle box jellyfish stings are known to cause Irukandji syndrome and Irukandji-like syndrome. Small carybdeid jellyfish, specifically Carukia barnesi are considered to be the main cause of Irukandji syndrome [11]. Other Australian carybdeid jellyfish are Morbakka spp. and Morbakka fenneri [12, 13]. There are at least three unnamed species of single-tentacle box jellyfish known to cause injuries in Thailand. However, it is possible that other species can cause Irukandji-like syndrome with different clinical manifestations $[9,10,12,14]$.

A case definition of Irukandji syndrome has been described as a severe local and systemic reaction occurring after a Carukia barnesi box jellyfish sting involving exposed

Prof. Lakkana Thaikruea, Department of Community Medicine, Faculty of Medicine, Chiang Mai University, Thailand, tel: 66-53-945471 to 4,

mobile phone: 66-8-6-9204243, e-mail: lakkana.t@cmu.ac.th 
skin [11]. The symptoms and signs developed between 5 and 40 minutes after being stung. These were predominantly pain and autonomic disturbances, such as severe muscle pain, muscle cramps, vomiting, sweating, agitation, hypertension, and heart failure [15-17]. However, Irukandji syndrome definition is still widely misunderstood, including species causing the syndrome, toxins, and geographic distribution. There had been reports of box jellyfish envenomation presenting as Irukandji syndrome but with other different symptoms and signs [16, 18]. Irukandji-like syndrome is more unclear than Irukandji syndrome. The Irukandji-like syndrome case report that occurred in Victoria was in 1998 [19]. Irukandji-like syndrome was described as: "Other jellyfish species (Not Carukia barnesi) cause a similar but not necessarily identical symptom complex referred to as Irukandji-like syndrome" [16]. The first case report of Irukandji syndrome in Thailand was published in 2001, which should have been described as Irukandji-like syndrome [20]. Physicians rarely diagnosed Irukandji-like syndrome in Thailand due to limited knowledge and inadequate laboratory facilities [8]. There is little known about the clinical manifestations of Irukandji-like syndrome caused by single-tentacle box jellyfish found in Thailand. The findings will be useful for diagnosis, health care, surveillance, and prevention and control measures. This study aimed to describe Irukandji-like syndrome associated with cases of stinging by single-tentacle box jellyfish found in Thailand.

\section{MATERIALS AND METHODS}

A medical epidemiologist from the Ministry of Public Health (MOPH) and one from the Faculty of Medicine of Chiang Mai University (author) started to investigate whether lethal jellyfish existed in Thailand as long ago as 2007. They established ad hoc toxic jellyfish surveillance in 2009, which later became the National surveillance system. They formed a steering team and invited officers from the Ministry of Natural Resources and Environment to join. This steering team established three toxic jellyfish networks, specifically task forces, experts, and communities. The initial members included Divers Alert Network, experts and health personnel in Thailand and a journalist and experts from universities in Australia. The membership expanded to stakeholders such as resort/hotel managers/owners, divers, speed boat/long-tail boat groups and biologists in order to detect the cases, build knowledge and collaborate regarding strategy. In the period from 2007 to 2019, all cases of stinging by single-tentacle box jellyfish resulting in collapse, hospital attendance or death were investigated. The investigations were conducted under the government service policy of emergency public health problems. These cases were included in this study. Data included details of the incident, physical examination, photographs of the wound, description of the box jellyfish, and a sample (if available) of the tentacle of the box jellyfish using Vacuum Sticky Tape for Identification of Toxic Jellyfish Class $[5,10]$. The same technique was used for the collection and transfer of the tentacle of the single-tentacle box jellyfish from the incident place and for nematocyst identification to determine the class of box jellyfish $[5,10]$.

Descriptive analyses included proportion, mean \pm standard deviation (SD), or median (minimum, maximum) depending on data distribution. Data management and analyses were performed using Epi Info for Windows version 7 (Centres for Disease Control and Prevention, Atlanta, GA).

\section{RESULTS}

\section{INCIDENT BY PERSON, TIME AND PLACE}

During the period from 2007 to 2019, 19 cases of stinging by single-tentacle box jellyfish were detected and investigated by the surveillance system. No fatalities were reported. The majority of cases were male (68.2\%), with a median age of 35.0 years (range $6.0-60.0$ years), tourists $(52.3 \%)$, and Thai nationality $(52.3 \%)$. The places where the incidents occurred were located along both coasts of Thailand. The three provinces with the highest reported incidence were Surat Thani (52.6\%), Krabi (15.8\%), and Trang (10.5\%). The median number of incidences was 3 cases per year and the highest incidence was in 2016 (26.3\%). About 31.6\% of cases were received appropriate first aid (Vinegar poured on the wounds immediately for at least $30 \mathrm{~s}$; Table 1). Among 19 cases, 6 of them could identify species group of single-tentacle box jellyfish. All of them were Morbakka spp. (31.6\%). All cases had severe wound pain with immediate systemic reaction.

\section{CLINICAL MANIFESTATIONS}

Clinical manifestations of Irukandji-like syndrome were categorised into three groups. These were severe wound pain with immediate systemic reaction (66.7\%), moderate wound pain with gradually systemic reaction (16.7\%), and moderate wound pain with immediate systemic reaction after physical or chemical trigger (16.7\%), such as rubbing with sand, taking a bath in freshwater, and rubbing with soap. The duration between the time of sting and pain development varied from a few seconds to a few minutes. The description of pain occurring when being stung included excruciating, burning, electric shock, and heart pain. The pain experienced during the systemic reaction included: headache, back pain, muscle pain, joint pain, abdominal pain, and body aches similar to being stabbed by knives (sharp jabbing pain). The pain was not constant but came in looping waves that increased for a few hours and lasted for 1 to 2 days.

The top five common symptoms and signs were pain (100.0\%), high blood pressure (100.0\%), palpitations 
Table 1. Incident by the person, time, place in 19 Irukandji-like syndrome cases

\begin{tabular}{|c|c|c|}
\hline Characteristics & Frequency & Per cent \\
\hline \multicolumn{3}{|l|}{ Occupation } \\
\hline Tourist & 10 & 52.3 \\
\hline Fisherman & 4 & 21.1 \\
\hline Jet ski rider & 1 & 5.3 \\
\hline Divemaster & 1 & 5.3 \\
\hline Civil servant & 1 & 5.3 \\
\hline Volunteer & 1 & 5.3 \\
\hline Employee & 1 & 5.3 \\
\hline \multicolumn{3}{|l|}{ Nationality } \\
\hline Thai & 10 & 52.3 \\
\hline British & 3 & 15.8 \\
\hline American & 2 & 10.5 \\
\hline Finnish & 1 & 5.5 \\
\hline French & 1 & 5.5 \\
\hline Dutch & 1 & 5.5 \\
\hline Burmese & 1 & 5.5 \\
\hline \multicolumn{3}{|l|}{ First aid } \\
\hline Appropriate vinegar pouring & 6 & 31.6 \\
\hline No first aid & 6 & 31.6 \\
\hline Inappropriate first aid & 6 & 31.6 \\
\hline Late vinegar pouring & 1 & 5.2 \\
\hline \multicolumn{3}{|l|}{ Year of occurrence } \\
\hline 2007 & 2 & 10.5 \\
\hline 2008 & 2 & 10.5 \\
\hline 2014 & 3 & 15.8 \\
\hline 2015 & 1 & 5.5 \\
\hline 2016 & 5 & 26.3 \\
\hline 2017 & 3 & 15.8 \\
\hline 2019 & 3 & 15.8 \\
\hline \multicolumn{3}{|l|}{ Province } \\
\hline Surat Thani & 10 & 52.6 \\
\hline Krabi & 3 & 15.8 \\
\hline Trang & 2 & 10.5 \\
\hline Phuket & 1 & 5.3 \\
\hline Petchburi & 1 & 5.3 \\
\hline Stun & 1 & 5.3 \\
\hline Chonburi & 1 & 5.3 \\
\hline
\end{tabular}

(86.7\%), respiratory distress (52.6\%), and near collapse/collapse (31.6\%) (Table 2).

The systemic reaction presented in waves of mild to severe symptoms and signs, including burning pain, headache,
Table 2. Symptoms and signs in nineteen Irukandji-like syndrome cases

\begin{tabular}{lll}
\hline Symptoms and signs & Frequency & Per cent \\
\hline Pain & 19 & 100.0 \\
High blood pressure* & 14 & 100.0 \\
Palpitations** & 13 & 86.7 \\
Respiratory distress & 10 & 52.6 \\
Near collapse/collapse & 6 & 31.6 \\
Fatigue & 5 & 26.3 \\
Nausea or vomiting & 5 & 26.3 \\
Anxiety/agitation & 4 & 21.0 \\
Abdominal cramp & 4 & 21.0 \\
Sweating & 2 & 10.5 \\
*Excluded 5 cases with no record $* *$ Excluded 4 cases with no record
\end{tabular}

*Excluded 5 cases with no record; **Excluded 4 cases with no record

body aches, back pain, muscle pain, joint pain, abdominal cramps, nausea, vomiting, fatigue, paralysed limbs, respiratory distress, palpitation, high blood pressure, sweating, anxiety, and agitation.

\section{WOUND CHARACTERISTICS}

All cases had tentacle marks. In all cases, except four with healing wounds when undergoing a physical examination, the marks from the tentacles appeared similar in appearance to the caterpillar tracks of tanks which have articulated steel bands passing around the wheels with repeated interspersion by normal tissue (Fig. 1). The width of the brown erythematous tentacle marks varied (Fig. 1A, B).

Three cases had blisters within 1 day after being stung. One case had sweat on the right hand which had been in contact with box jellyfish tentacles 18.5 hours after being stung and lasted for 2 days (Fig. 2).

\section{DISCUSSION}

The actual incidence of cases of Irukandji-like syndrome might be higher than that recorded in this paper. Two of the reasons for this are that health personnel might give a misdiagnosis and cases with mild symptoms might not go to a doctor [7, 14]. However, this study emphasized moderate to severe cases in order to reduce false positive case. Although the majority of the cases are tourists, fishermen are another vulnerable population that needs consideration and they are active all year round. Many Thais believe that Caucasian people have a hypersensitivity to jellyfish not Thai people $[7,14]$. This study found that more than half of the cases were Thai nationals and had clinical manifestation that indicated a non-allergic response to the toxin $[2,7,14$, 21]. The highest number of cases was in the Surat Thani Province in the Gulf of Thailand. Although fatal cases stung by multiple-tentacle box jellyfish were found in this province, 

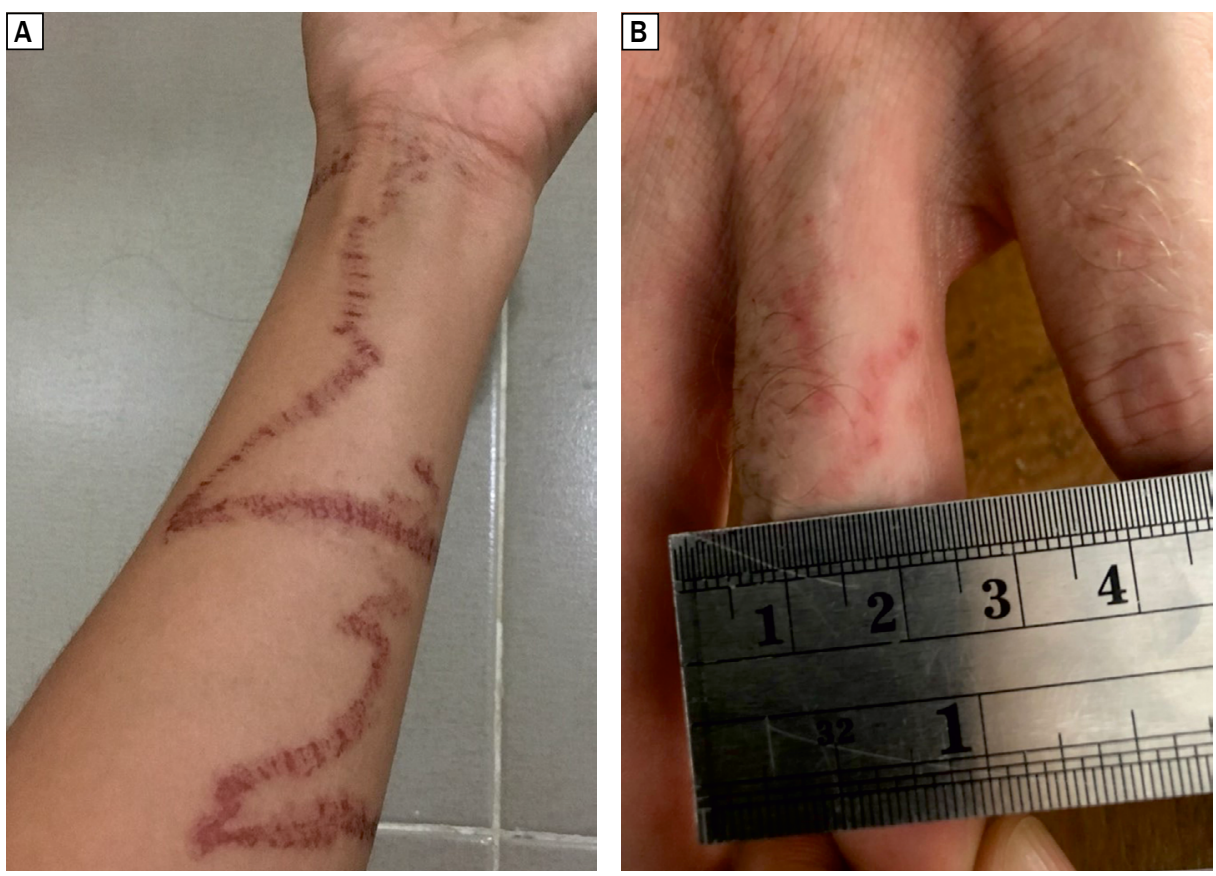

Figure 1. The width of the tentacle marks varied; A. Erythematous brownish colour on the second day after being stung by Morbakka spp.; B. Erythematous colour on the second day after being stung by other species on the left ring finger (image source: Cases)

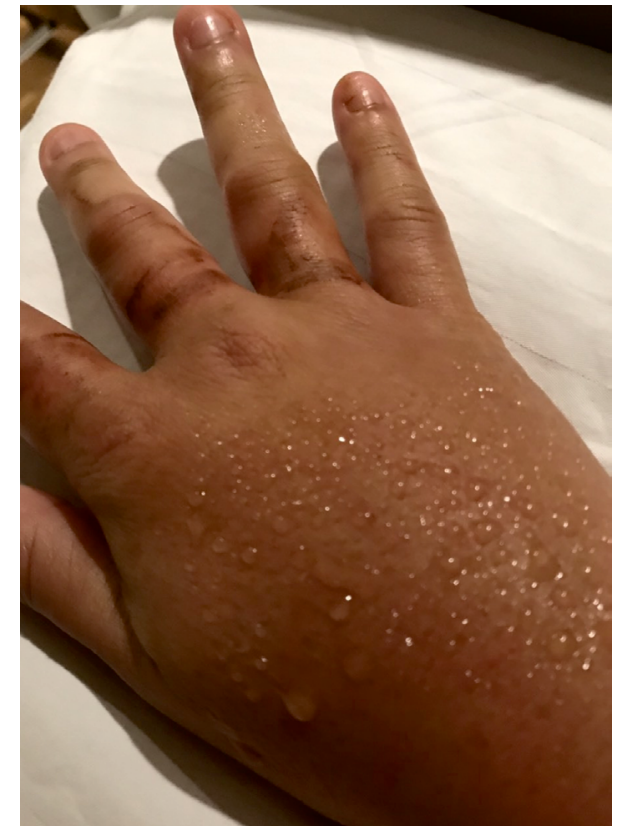

Figure 2. Sweat appeared on the right hand which had been in contact with box jellyfish tentacles 18.5 hours after being stung (image source: Case)

there was no report of a fatal case stung by single-tentacle box jellyfish $[4,7,8,14]$.

Appropriate first aid for stings by box jellyfish found in Thailand is pouring vinegar (4-6\% acetic acid) for at least 30 seconds continuously on the wound immediately after contact with the tentacle (except for stings in the eye) [6-8,
22-24]. In more recent years, stings were treated with vinegar as the intervention measures had been launched. Based on the author's experiences, severe cases of being stung by box jellyfish who had late vinegar poured on the wounds (as long as 10 to $15 \mathrm{~min}$ ) after the events survived. Thus, vinegar pouring is recommended for first aid $[7,8$, 22-25]. The steering team contributed the results from researches, surveillance, and outbreak investigations to policymakers, which led to prevention and control measures, including vinegar stations and educational warning signs being installed at the beaches $[3,5,7]$.

This study showed that the majority of cases experienced severe wound pain with an immediate systemic reaction, while Irukandji syndrome sufferers usually had mild to moderate wound pain with a gradual systemic reaction about 5 to 60 minutes after being stung [16, 26]. Some cases had moderate wound pain with an immediate systemic reaction after physical or chemical triggers (such as rubbing sand or soap into the wound, taking a bath with freshwater). The possible explanation of the immediate systemic reaction might be due to more toxin entering the system due to physical or chemical triggers. It is worth noting that the pain occurring when being stung differed from the pain occurring during the systemic reaction. The pain occurs when being stung was excruciating or burning pain at the wounds, felt like an electric shock, and rapidly expanded to heart pain. While the pain occurring during the systemic reaction was back pain, muscle pain, joint pain, abdominal pain, and body aches. These pains were not constant but 
came in waves which increased in a few hours and lasted for 1 to 2 days. Therefore, a clear case definition can help in diagnosis, health care, and advice patients. The clinical manifestations of multiple-tentacle box jellyfish sting found in Thailand usually present with immediate severe wound pain following the stings and do not have waves of increasing pain and loops of systemic reaction $[7,8,14,27]$.

The tentacle marks found in this study appeared as caterpillar tracks of the tank that have articulated steel bands passing around the wheels, which is similar to that of multiple-tentacle box jellyfish [7, 8, 14, 24, 27]. According to the context of Thailand, Thaikruea et al. [7, 24] used caterpillar track appearance in defining Thai language term as "Teen-ta-kab" for risk communication because it is more understandable than other professional's terms (i.e. "Frostladder-like", "Step ladder-like", or "Ladder-like transverse band"). The tentacle marks caused by single-tentacle box jellyfish stings often cover a smaller area of skin than those caused by multiple-tentacle box jellyfish stings. However, it is difficult to distinguish between a few tentacle marks caused by multiple-tentacle box jellyfish stings and tentacle marks caused by single-tentacle box jellyfish stings. Thus, a history of exposure and clinical manifestation is important information for diagnosis [27]. Another interesting wound characteristic was blistering. Blisters were reported among severe cases stung by multiple-tentacle box jellyfish found in Thailand $[14,27]$. The blisters in these cases developed within 24 to 48 hours among cases with massive stings or incorrect first aid. In some cases, blisters developed on the $4^{\text {th }}$ or $5^{\text {th }}$ day after being stung $[14,27]$. In this study, 3 cases had blisters within 24 hours. Possible explanations were probably high doses of toxin due to massive stings ( 1 case) and incorrect first aid which increased nematocyst firing ( 2 cases). This finding is useful because blister has been reported only in severe wound complications among cases involving stings by multiple-tentacle box jellyfish [6]. However, only 1 case had deep dermal necrosis and needed wound debridement for about 2 months in this study. Another study reported a delayed development of dermal necrosis in a girl with one of these stings [28]. One case had sweating localised on the skin area which had been in contact with the tentacle of a single-tentacle box jellyfish. Interestingly this sign has not been reported in any cases involving stings by both single and multiple-tentacle box jellyfish found in Thailand.

This study found that the systemic reactions presented in waves of mild to severe symptoms and signs. These symptoms and signs were similar to those of Irukandji syndrome $[16,26,29]$. However, no cases report any feelings of impending doom. They were more likely to experience anxiety or agitation. The common symptoms and signs can be used for determination and diagnosis. In this study, the top five most common symptoms and signs were pain, high blood pressure, palpitation, near collapse/collapse, and fatigue. Although less common, nausea and vomiting were found in cases involving stings by multiple-tentacle box jellyfish found in Thailand $[7,8,14,27]$. Based on the author's experiences, this nausea and vomiting symptoms without taking a history of jellyfish contact and no/unnoticed tentacle mark may lead to misdiagnosis of other possible illnesses such as food poisoning and appendicitis among children.

According to the findings of symptoms and signs related to cardiovascular and respiratory systems, monitoring of vital signs is recommended for treatment assessment and progression. It is worth noting that all cases involving stings by Morbakka spp. had severe wound pain with immediate systemic reaction. Fenner et al. [13] reported the first case involving a sting by Morbakka spp. which had similar symptoms and signs to the findings in this study. Based on toxic jellyfish networks in the surveillance system, Morbakka spp. were found in diving spots in the deeper areas of the sea. In recent years, they have also been found in shallow water near the beach. An outbreak investigation team from MOPH reported Morbakka spp. in August 2003 [8]. A new species Morbakka fenneri was discovered and identified as a species of Irukandji jellyfish [12]. However, the actual species of Morbakka spp. found in Thailand have not yet been identified. To date, they are named as Morbakka spp.A, Morbakka spp.B, and Morbakka spp.C [9]. Different clinical manifestations support the possibility that more than one species of single-tentacle box jellyfish found in Thailand can cause Irukandji-like syndrome. Further studies should be conducted to prove this observation. The location and species of single-tentacle box jellyfish are useful in distinguishing between Irukandji syndrome and Irukandji-like syndrome.

There were some limitations to this study, the species of single-tentacle box jellyfish could not be identified in some cases due to the practical difficulty in catching the jellyfish. Also, the setting up of laboratory facilities for research has only occurred recently. The numbers of cases might be somewhat underestimated due to misdiagnosis or undetected by surveillance in earlier years when there was a lack of Irukandji-like syndrome knowledge. However, the number might have little effect on the findings of this study because the toxic jellyfish networks of the surveillance system cover both coasts of Thailand and communities and stakeholders engaged in the surveillance and interventions $[2,3,5,7,10]$.

\section{CONCLUSIONS}

This is the largest study of Irukandji-like syndrome cases involving stings by single-tentacle box jellyfish in Thailand and the clinical manifestations differ from those of 
multiple-tentacle box jellyfish stings. The different clinical manifestations in these cases might be caused by different species of single-tentacle box jellyfish. Clear clinical manifestation is particularly important in this context that communities play a major role in early warning and rapid response. The practical definition will be useful for medical care, surveillance system, and prevention and control measures.

\section{ACKNOWLEDGEMENTS}

I would like to express my thanks to Chiang Mai University and the Faculty of Medicine for management support. I would like to thank Dr. Pitikhun Setapura, Dr. Potjaman Siriariyaporn, Miss Hataya Kanjanasombut and Miss Kannika Monpangtiem (Epidemiology Division, Ministry of Public Health) for information

\section{REFERENCES}

1. Thaikruea L. A case study of Thailand's first toxic jellyfish networks and knowledge establishment. In: Socially-engaged scholarship. Bangkok, Thailand: Civil Society Engagement Thailand. 2016: 26-29.

2. Thaikruea L. A decade of fighting the lethal jellyfish health problem: lesson learned. Proceedings of the 4th Asiaengage Regional Conference; 2018 Nov 26-28; Chiang Mai, Thailand. Chiang Mai: Chiang Mai University. 2018: 95.

3. Thaikruea L, Siriariyaporn P. Surveillance system and prevention for injuries and deaths. In: Injuries and Deaths Caused by Toxic Jellyfish: Surveillance, Prevention, and Treatment. Chiang Mai: Faculty of Medicine of Chiang Mai University. 2018: 177-240.

4. Sucharitakul P, Chomdej S, Achalawitkun T, et al. Chirodropid box jellyfish in the Gulf of Thailand. Marine Biodiversity. 2018; 49(3): 1247-1252, doi: 10.1007/s12526-018-0887-4.

5. Thaikruea L, Santidherakul S. The public health impact of a new simple practical technique for collection and transfer of toxic jellyfish specimens and for nematocyst identification. J Public Health Policy. 2018; 39(2): 143-155, doi: 10.1057/s41271-017-0112-x, indexed in Pubmed: 29217831.

6. Thaikruea L, Siriariyaporn P. Severe dermatonecrotic toxin and wound complications associated with box jellyfish stings 2008-2013. J Wound Ostomy Continence Nurs. 2015; 42(6): 599-604, doi: 10.1097/WON.0000000000000190, indexed in Pubmed: 26528872.

7. Thaikruea L, Siriariyaporn P. The magnitude of severe box jellyfish cases on Koh Samui and Koh Pha-ngan in the Gulf of Thailand. BMC Res Notes. 2016; 9: 108, doi: 10.1186/s13104-016-1931-8, indexed in Pubmed: 26888067.

8. Thaikruea L, Siriariyaporn P, Wutthanarungsan R, et al. Review of fatal and severe cases of box jellyfish envenomation in Thailand. Asia Pac J Public Health. 2015; 27(2): NP1639-NP1651, doi: 10.1177/1010539512448210, indexed in Pubmed: 22743852.

9. Thaikruea L, Siriariyaporn P. Jellyfish. In: Injuries and Deaths Caused by Toxic Jellyfish: Surveillance, Prevention, and Treatment. Chiang Mai: Faculty of Medicine of Chiang Mai University. 2018: 1-28.

10. Thaikruea L, Siriariyaporn P. Nematocyst and identification technique. In: Injuries and Deaths Caused by Toxic Jellyfish: Surveillance, Prevention, and Treatment. Chiang Mai: Faculty of Medicine of Chiang Mai University. 2018: 157-176.

11. Barnes $\mathrm{JH}$. Cause and effect in irukandji stingings. Med J Australia. 1964; 1(24): 897-904, doi: 10.5694/j.1326-5377.1964.tb114424.x.

12. Gershwin, L. Morbakka fenneri, a new genus and species of Irukandji jellyfish (Cnidaria: Cubozoa). Mem QueensI Mus. 2008; 54(1): 23-33.
13. Fenner PJ, Fitzpatrick PF, Hartwick RJ, et al. "Morbakka", another cubomedusan. Med J Aust. 1985; 143(12-13): 550-555, indexed in Pubmed: 2870414.

14. Thaikruea L, Siriariyaporn P. Situation of injuries and deaths. In: Thaikruea L, editor. Injuries and Deaths Caused by Toxic Jellyfish: Surveillance, Prevention, and Treatment. Chiang Mai: Faculty of Medicine of Chiang Mai University. 2018: 29-84.

15. Fenner P, Harrison S. Irukandji and Chironex fleckeri jellyfish envenomation in tropical Australia. Wilderness \& Environmental Medicine. 2000; 11(4): 233-240, doi: 10.1580/1080-6032(2000)011[0233:iacfje]2.3.co;2.

16. Grady JD, Burnett JW. Irukandji-like syndrome in South Florida divers. Ann Emerg Med. 2003; 42(6): 763-766, doi: 10.1016/s01960644(03)00513-4, indexed in Pubmed: 14634600.

17. Huynh TT, Seymour J, Pereira P, et al. Severity of Irukandji syndrome and nematocyst identification from skin scrapings. Med J Aust. 2003; 178(1): 38-41, indexed in Pubmed: 12492390.

18. Burnett JW, Weinrich D, Williamson JA, et al. Autonomic neurotoxicity of jellyfish and marine animal venoms. Clin Auton Res. 1998; 8(2): 125-130, doi: 10.1007/BF02267823, indexed in Pubmed: 9613803.

19. Cheng AC, Winkel KD, Hawdon GM, et al. Irukandji-like syndrome in Victoria. Aust N Z J Med. 1999; 29(6): 835, doi: 10.1111/j.14455994.1999.tb00797.x, indexed in Pubmed: 10677135.

20. Pender AM, Winkel KD, Ligthelm RJ. A Probable Case of Irukandji Syndrome in Thailand. J Travel Med. 2006; 13(4): 240-243, doi: 10.1111/j.1708-8305.2006.00041.x.

21. Thaikruea L, Siriariyaporn $P$, Wutthanarungsanm $R$, et al. Toxic jellyfish situation in Thailand. Chiang Mai Med J. 2012; 51(4): 93-102.

22. Cegolon L, Heymann WC, Lange JH, et al. Jellyfish stings and their management: a review. Mar Drugs. 2013; 11(2): 523-550, doi: 10.3390/md11020523, indexed in Pubmed: 23434796.

23. Thaikruea L, Siriariyaporn P. First aid and treatment. In: Injuries and Deaths Caused by Toxic Jellyfish: Surveillance, Prevention, and Treatment. Chiang Mai: Faculty of Medicine of Chiang Mai University. 2018: 115-156.

24. Thaikruea L, Leelarasamee A. Which First Aid Treatment Is Appropriate for a Bizarre Skin Lesion and Cardiovascular Collapse after Swimming in the Sea? J Med Assoc Thai. 2018; 101(8): 1143-1144.

25. Markenson D, Ferguson JD, Chameides L, et al. First Aid Chapter Collaborators. Part 13: First aid: 2010 American Heart Association and American Red Cross International Consensus on First Aid Science With Treatment Recommendations. Circulation. 2010; 122(16 Suppl 2): S582-S605, doi: 10.1161/CIRCULATIONAHA.110.971168, indexed in Pubmed: 20956261.

26. Fenner PJ, Hadok JC. Fatal envenomation by jellyfish causing Irukandji syndrome. Med J Aust. 2002; 177(7): 362-363, doi: 10.5694/ j.1326-5377.2002.tb04838.x, indexed in Pubmed: 12358578.

27. Thaikruea L, Siriariyaporn P. Diagnoses for injuries and deaths. In: Injuries and Deaths Caused by Toxic Jellyfish: Surveillance, Prevention, and Treatment. Chiang Mai: Faculty of Medicine of Chiang Mai University. 2018: 85-114.

28. Desax-Willer D, Krebs T, Christen S. Delayed deep dermal necrosis after jellyfish sting in a 4-year-old female infant. Case Reports Plast Surg Hand Surg. 2018; 5(1): 75-79, doi: 10.1080/23320885.2018.1533407, indexed in Pubmed: 30397636.

29. Nickson CP, Waugh EB, Jacups SP, et al. Irukandji syndrome case series from Australia's Tropical Northern Territory. Ann Emerg Med. 2009; 54(3): 395-403, doi: 10.1016/j.annemergmed.2009.03.022, indexed in Pubmed: 19409658. 